\title{
A GENERAL THEORY OF SURFACES AND CONJUGATE NETS
}

\author{
BY \\ V. G. GROVE
}

1. Introduction. The object of this paper is to present a general method of studying nondevelopable surfaces and conjugate nets from a projective point of view. The method used is that of tensors. An earlier paper [8](1) initiated the study we are attempting. A still earlier paper [6] presented a similar theory from a non-tensor point of view, but certain restrictions inherent to the method caused considerable loss of generality. The present study removes these restrictions.

Covariant differentiation is based on a connection arising naturally in the theory rather than on Christoffel symbols derived from a quadratic form. The components of this connection reduce to the Christoffel symbols based on the metric tensor if the geometry is specialized to that on a surface immersed in euclidean space of three dimensions. An easy direct method is therefore available for the study, from the metric point of view, of geometric entities commonly considered in projective geometry. The final section is devoted to these considerations.

In the discussion of any particular geometric entity, it is usually desirable to reduce the forms inherent to the discussion to suitable canonical forms. However if it is desired to study two or more disparate entities simultaneously, there is considerable labor involved in relating the canonical forms commonly associated with their study. For this reason we have delayed reducing our forms to a canonical form until $\$ 5$, leaving all formulas in unspecialized parameters.

Green showed [3] that the invariants (and covariants) of a surface expressed in terms of the asymptotic parameters may be expressed essentially in terms of arbitrary non-conjugate parameters without preliminary integrations. He assumed that the necessary integrations have been performed, then, by changing the parameters, computed the invariants of the surface in the new non-conjugate representation. In this paper we compute the invariants and covariants of a surface (or conjugate net) directly in terms of arbitrary parameters. The tensor notation makes these computations relatively easy.

Let the homogeneous projective coordinates $\left(x^{1}, x^{2}, x^{3}, x^{4}\right)$ of a point in a projective space of three dimensions be given as analytic functions of two parameters $u^{1}, u^{2}$. Denote by $S$ the surface generated by $x$. Let $\left(y^{1}, y^{2}, y^{3}, y^{4}\right)$

Presented to the Society, September 13, 1943; received by the editors November 1, 1943.

(1) Numbers in brackets refer to the references cited at the end of the paper. 
be the coordinates of a point $y$ not in the tangent plane to $S$ at $x$. The pairs of functions $x, y$ satisfy a system of differential equations of the form

$$
\begin{aligned}
\partial^{2} x / \partial u^{\alpha} \partial u^{\beta} & =L_{\alpha \beta}^{\rho} \partial x / \partial u^{p}+P_{\alpha \beta} x+D_{\alpha \beta} y, \\
\partial y / \partial u^{\alpha} & =M_{\alpha}^{\rho} \partial x / \partial u^{\rho}+Q_{\alpha} x+E_{\alpha} y,
\end{aligned}
$$

repeated indices indicating summation.

Under the transformation

$$
u^{\alpha}=u^{\alpha}\left(\bar{u}^{1}, \bar{u}^{2}\right), \quad J=\left|\partial u^{\alpha} / \partial \bar{u}^{\beta}\right| \neq 0
$$

the coefficients $L_{\alpha \beta}^{\rho}$ in (1.1) transform according to the law of transformation:

$$
\bar{L}_{\alpha \beta}^{\rho}=\left(L_{\mu \sigma}^{\lambda} \frac{\partial u^{\mu}}{\partial \bar{u}^{\alpha}} \frac{\partial u^{\sigma}}{\partial \bar{u}^{\beta}}+\frac{\partial^{2} u^{\lambda}}{\partial \bar{u}^{\alpha} \partial \bar{u}^{\beta}}\right) \frac{\partial \bar{u}^{\rho}}{\partial u^{\lambda}} .
$$

These functions may therefore be used as a basis for covariant differentiation. If covariant differentiation with respect to the connection $L_{\alpha \beta}^{p}$ be denoted by a comma, we may write equations (1.1) in the form

$$
\begin{aligned}
x_{, \alpha \beta} & =P_{\alpha \beta} x+D_{\alpha \beta} y, \\
y_{, \alpha} & =M_{\alpha}^{\rho} x_{, \rho}+Q_{\alpha} x+E_{\alpha} y .
\end{aligned}
$$

The integrability conditions of system (1.2) may be written in the form

$$
\begin{aligned}
P_{\alpha \beta, \gamma}-P_{\alpha \gamma, \beta} & =Q_{\beta} D_{\alpha \gamma}-Q_{\gamma} D_{\alpha \beta}, \\
D_{\alpha \beta, \gamma}-D_{\alpha \gamma, \beta} & =E_{\beta} D_{\alpha \gamma}-E_{\gamma} D_{\alpha \beta}, \\
M_{\alpha, \beta}^{\rho}-M_{\beta, \alpha}^{\rho} & =\delta_{\alpha}^{\rho} Q_{\beta}-\delta_{\beta}^{\rho} Q_{\alpha}+E_{\beta} M_{\alpha}^{\rho}-E_{\alpha} M_{\beta}^{\rho}, \\
Q_{\alpha, \beta}-Q_{\beta, \alpha} & =E_{\beta} Q_{\alpha}-E_{\alpha} Q_{\beta}+P_{\alpha \rho} M_{\beta}^{\rho}-P_{\beta \rho} M_{\alpha,}^{\rho}, \\
R_{\alpha \beta \gamma}^{\rho}+P_{\alpha \gamma} \delta_{\beta}^{\rho}-P_{\alpha \beta \delta_{\gamma}}^{\rho} & =D_{\alpha \beta} M_{\gamma}^{\rho}-D_{\alpha \gamma} M_{\beta}^{\rho}=P_{\alpha \beta \gamma}^{\rho}, \\
E_{\alpha, \beta}-E_{\beta, \alpha} & =D_{\alpha \rho} M_{\beta}^{\rho}-D_{\beta \rho} M_{\alpha}^{\rho}=P_{\rho \alpha \beta}^{\rho}=R_{\rho \alpha \beta .}^{\rho} .
\end{aligned}
$$

Let $z$ be a focal point on the line $l_{1}$ joining the points $x$ and $y$, and let $u^{\alpha}=u^{\alpha}(t)$ be the parametric equations of the curves on $S$ corresponding to the developables of the congruence $\Gamma_{1}$ of lines $l_{1}$. The general coordinates of $z$ are expressible in the form

$$
z=y-\phi x .
$$

The parameter $\phi$ and the differentials $d u^{\alpha}$ satisfy the respective equations

$$
\begin{aligned}
\phi^{2}-M_{\rho}^{\rho} \phi+M & =0, & M=\left|M_{\beta}^{\alpha}\right|, \\
M_{\sigma}^{\rho} I_{\rho \lambda} d u^{\alpha} d u^{\lambda} & =0, &
\end{aligned}
$$

wherein

$$
I_{11}=0, I_{12}=(-D)^{1 / 2}, I_{21}=-(-D)^{1 / 2}, I_{22}=0, D=\left|D_{\alpha \beta}\right| \neq 0
$$


The asymptotic curves on $S$ are defined by the differential equation

$$
D_{\rho \sigma} d u^{\rho} d u^{\sigma}=0 .
$$

The harmonic invariant of the forms appearing in the left members of (1.7) and the last of (1.5) may be written in the form

$$
M_{\beta}^{\rho} D_{\rho \alpha}-M_{\alpha}^{\rho} D_{\rho \beta}=P_{\rho \alpha \beta}^{\rho} \text {. }
$$

Hence the congruence $\Gamma_{1}$ is conjugate to $S$ if and only if

$$
E_{\alpha, \beta}-E_{\beta, \alpha}=0 \text {. }
$$

Under the transformation

$$
y=\theta^{\rho} x_{, \rho}+\phi x+a \bar{y},
$$$$
a \neq 0,
$$

the system (1.3) transforms into the system

$$
\begin{aligned}
x_{i \alpha \beta} & =\bar{P}_{\alpha \beta} x+\bar{D}_{\alpha \beta} \bar{y}, \\
\bar{y}_{; \alpha} & =\bar{M}_{\alpha}^{\rho} x_{; \rho}+\bar{Q}_{\alpha} x+\bar{E}_{\alpha} \bar{y},
\end{aligned}
$$

wherein the semicolon denotes covariant differentiation with respect to the transformed connection

$$
\bar{L}_{\alpha \beta}^{\rho}=L_{\alpha \beta}^{\rho}+D_{\alpha \beta} \theta^{\rho} .
$$

The remaining coefficients of (1.10) useful to us are given by the formulas

$$
\begin{aligned}
\bar{P}_{\alpha \beta} & =P_{\alpha \beta}+\phi D_{\alpha \beta}, \quad \bar{D}_{\alpha \beta}=a D_{\alpha \beta}, \\
a \bar{M}_{\alpha}^{\rho} & =M_{\alpha}^{\rho}-\phi \delta_{\alpha}^{\rho}-\theta_{, \alpha}^{\rho}+\left(\bar{E}_{\alpha}+A_{, \alpha}\right) \theta^{\rho}, \\
\bar{E}_{\alpha} & =E_{\alpha}-\theta_{\alpha}-A_{, \alpha}, \quad A=\log a,
\end{aligned}
$$

wherein

$$
\theta_{\alpha}=D_{\alpha \rho} \theta^{\rho}
$$

It follows that the components $P_{\rho \alpha \beta}^{\rho}$ transform under (1.9) according to the law

$$
\bar{P}_{\rho \alpha \beta}^{\rho}=\left(E_{\alpha}-\theta_{\alpha}\right)_{, \beta}-\left(E_{\beta}-\theta_{\beta}\right)_{, \alpha} .
$$

Let $D^{\alpha \beta}$ be defined by the relation.

$$
D^{\alpha \rho} D_{\rho \beta}=\delta_{\beta}^{\alpha} \text {. }
$$

Now in the transformation (1.9) choose

$$
\theta^{\alpha}=\left(E_{\sigma}-\omega_{, \sigma}-A_{, \sigma}\right) D^{\alpha \sigma},
$$

wherein $\omega$ is an arbitrary differentiable function. Then the transform of $E_{\alpha}$ under (1.9) is given by the formula

$$
\bar{E}_{\alpha}=\omega_{, \alpha}
$$


The congruence $\Gamma_{1}$ of lines $\bar{l}_{1}$ joining $x, \bar{y}$ is conjugate to $S$. Hence we may without quadratures, and in any parametric representation whatever, find all congruences conjugate to a given surface. These congruences depend upon one arbitrary differentiable function of the arbitrary parameters on the surface.

From (1.5) the harmonic conjugate of $x$ with respect to the focal points on $l_{1}$ is the point $\zeta$ whose general coordinates are given by the expression

$$
\zeta=y+M_{p}^{p} x / 2 .
$$

We shall call this point the $K$-point of $x$ on the line $l_{1}$.

The curves on $S$ corresponding to the developables of the congruences $\Gamma_{2}$ of the lines $l_{2}$ joining the points $r_{1}, r_{2}$ whose coordinates are defined by the expression

$$
r_{\alpha}=x_{, \alpha}+\lambda_{\alpha} x
$$

are the integral curves of the differential equation

$$
\pi_{\mu \sigma} D^{\mu \mu} I_{\rho \lambda} d u^{\sigma} d u^{\lambda}=0,
$$

wherein

$$
\pi_{\alpha \beta}=P_{\alpha \beta}+\lambda_{\alpha, \beta}-\lambda_{\alpha} \lambda_{\beta} .
$$

The curves (1.12) form a conjugate net if and only if

$$
\pi_{\alpha \beta}=\pi_{\beta \alpha},
$$

that is, if and only if

$$
\lambda_{\alpha, \beta}-\lambda_{\beta, \alpha}=0 .
$$

Differentiating (1.11) covariantly one obtains

$$
r_{\alpha, \beta}-\lambda_{\alpha} r_{\beta}=\pi_{\alpha \beta} x+D_{\alpha \beta} y .
$$

If (1.13) holds, one obtains from (1.15) the equation

$$
\partial r_{1} / \partial u^{2}-\lambda_{2} r_{1}=\partial r_{2} / \partial u^{1}-\lambda_{1} r_{2} .
$$

An obvious geometrical interpretation may therefore be made for the condition (1.13).

Let $\Lambda$ be a differentiable, but otherwise arbitrary, function of $u^{1}, u^{2}$. If in (1.11) we let $\lambda_{\alpha}=\Lambda_{, \alpha}$ the condition (1.14) is satisfied. Hence the most general congruence harmonic to $S$ is the congruence of lines joining $r_{1}, r_{2}$ where

$$
r_{\alpha}=x_{, \alpha}+\Lambda_{, \alpha} x, \quad \alpha=1,2 .
$$

2. Reciprocal congruences. Let the roots of the quadratic (1.7) be written in the form

$$
d u^{1}: d u^{2}=A^{1}: A^{2}, \quad d u^{1}: d u^{2}=B^{1}: B^{2},
$$

and choose the proportionality factor for $A^{\alpha}, B^{\alpha}$ so that 


$$
A^{\alpha} B^{\beta}+B^{\alpha} A^{\beta}=2 D^{\alpha \beta} \text {. }
$$

If we let

$$
A_{\alpha}=D_{\alpha \rho} A^{p}, \quad B_{\alpha}=D_{\alpha \rho} B^{p},
$$

then

$$
A_{\alpha} B_{\beta}+B_{\alpha} A_{\beta}=2 D_{\alpha \beta}, \quad A_{\rho} B^{\rho}=A^{\rho} B_{\rho}=2 .
$$

It also follows that

$$
\begin{aligned}
& A_{\alpha} B_{\beta}-B_{\alpha} A_{\beta}=2 I_{\alpha \beta}, \\
& A^{\alpha} B^{\beta}-B^{\alpha} A^{\beta}=2 I^{\alpha \beta},
\end{aligned}
$$

wherein

$$
I^{11}=0, \quad I^{12}=(-D)^{1 / 2}, \quad I^{21}=-(-D)^{1 / 2}, \quad I^{22}=0 .
$$

We shall call the asymptotic curve whose tangent vector is $A^{\alpha}\left(B^{\alpha}\right)$ the $A$-curve ( $B$-curve) with similar appellations for the tangents to these curves. Consider now the points $X, Y$ lying respectively on the $A$-tangent and $B$ tangent. The coordinates of these points are of the form

$$
X,=A^{\rho} x_{, \rho}+A x, \quad Y=B^{\rho} x_{, \rho}+B x .
$$

As $x$ moves along the $B$ curve the point $X$ moves on a curve a point $X^{\prime}$ on whose tangent has coordinates given by the expression

$$
X^{\prime}=2 y+\left(A_{, \sigma}^{\rho} B^{\sigma}+A^{\prime} B^{\rho}+\lambda A^{\rho}\right) x_{, \rho}+\left(A_{, \sigma} B^{\sigma}+P_{\rho \sigma} A^{\rho} B^{\sigma}+\lambda A\right) x .
$$

In a similar manner we define a point $Y^{\prime}$ by the formula

$$
Y^{\prime}=2 y+\left(B_{, \sigma}^{\rho} A^{\sigma}+B A^{\rho}+\mu B^{\rho}\right) x_{, \rho}+\left(B_{, \sigma} A^{\sigma}+P_{\rho \sigma} A^{\rho} B^{\sigma}+\mu B\right) x .
$$

The points $X^{\prime}, Y^{\prime}$ and $x$ are collinear if and only if $\lambda, \mu, A, B$ satisfy the equation

$$
\lambda A^{\rho}+A B^{\rho}+A_{,{ }_{\sigma}}^{\rho} B^{\sigma}=\mu B^{\rho}+B A^{\rho}+B_{,{ }_{\sigma}} A^{\sigma} .
$$

Hence

$$
\begin{aligned}
& \lambda=\left(B_{\rho} / 2\right)\left(B_{, \sigma}^{\rho} A^{\sigma}-A_{, \sigma}^{\rho} B^{\sigma}\right)+B=B_{\rho, \sigma} I^{\rho \sigma}+B, \\
& \mu=\left(A_{\rho} / 2\right)\left(A_{,{ }_{\sigma}}^{\rho} B^{\sigma}-B_{, \sigma}^{\rho} A^{\sigma}\right)+A=A_{\rho, \sigma} I^{\sigma \rho}+A .
\end{aligned}
$$

For this choice of $\lambda, \mu$ a point $Z$ on the line joining $x, X^{\prime}$ (or $x, Y^{\prime}$ ) has coordinates given by the formula

$$
Z=y+F^{\rho} x, \rho / 2
$$

wherein $F^{\alpha}$ is defined by either of the equivalent expressions

$$
F^{\alpha}=A_{, \sigma}^{\alpha} B^{\sigma}+\left(A^{\alpha} B_{\lambda} / 2\right)\left(B_{,{ }_{\sigma}}^{\lambda} A^{\sigma}-A_{,{ }_{\sigma}}^{\lambda} B^{\sigma}\right)+B A^{\alpha}+A B^{\alpha},
$$




$$
F^{\alpha}=B_{, \sigma}^{\alpha} A^{\sigma}+\left(B^{\alpha} A_{\lambda} / 2\right)\left(A_{,{ }_{\sigma}}^{\lambda} B^{\sigma}-B_{, \sigma}^{\lambda} A^{\sigma}\right)+A B^{\alpha}+B A^{\alpha} .
$$

Combining these expressions and making use of (2.1) and (2.2) we may write $F^{\alpha}$ in the form

$$
F^{\alpha}=-T^{\alpha}+A B^{\alpha}+B A^{\alpha}
$$

wherein

$$
\begin{aligned}
T^{\alpha} & =D^{\rho \sigma} T_{\rho \sigma}^{\alpha}, \\
T_{\rho \sigma}^{\alpha} & =D^{\alpha \lambda}\left(D_{\rho \lambda, \sigma}+D_{\sigma \lambda, \rho}-D_{\rho \sigma, \lambda}\right) / 2 .
\end{aligned}
$$

There exists a unique line $l_{1}$ through $x$ and intersecting the tangents to the loci of $X$ and $Y$ as $x$ moves respectively along the $B$-curve and $A$-curve on $S$. This line is determined by $x$ and the point $Z$ whose coordinates are given by (2.5). The line $l_{1}$ so defined and the line $l_{2}$ determined by $X$ and $Y$ are of course reciprocal lines [5].

Conversely the line $l_{2}$ is the reciprocal of the line $l_{1}$ joining $x$ to $Z$ defined by the expression

$$
Z=y-\theta^{\rho} x, \rho
$$

if $A$ and $B$ are so chosen that

$$
\theta^{\rho}=\left[T^{\rho}-\left(A B^{\rho}+B A^{p}\right)\right] / 2 .
$$

Hence the line $l_{2}$ joining the points $X, Y$ defined by the expressions

$$
\begin{aligned}
X & =A^{\rho}\left[x_{, \rho}+\left(T_{\rho}-2 \theta_{\rho}\right) x / 2\right], \\
Y & =B^{\rho}\left[x_{, \rho}+\left(T_{\rho}-2 \theta_{\rho}\right) x / 2\right]
\end{aligned}
$$

is the reciprocal of the line $l_{1}$ joining $x$ to the point $Z$ defined by (2.6). This line $l_{2}$ intersects the parametric tangents in the points $r_{1}, r_{2}$ whose coordinates are given by the expression

$$
r_{\alpha}=x_{, \alpha}+\left(T_{\alpha}-2 \theta_{\alpha}\right) x / 2 .
$$

Suppose the congruence $\Gamma_{2}$ of lines $l_{2}$ is harmonic to $S$. Then from (1.16) we find that $l_{2}$ intersects the asymptotic tangents in the points $X, Y$ whose coordinates are given by the expressions

$$
X=A^{\rho}\left(x_{, \rho}+\Lambda_{, \rho} x\right), \quad Y=B^{p}\left(x_{, \rho}+\Lambda_{, \rho} x\right) .
$$

The reciprocal polar $l_{1}$ of $l_{2}$ joins $x$ to the point $Z$ whose coordinates are

$$
Z=y-\left(T^{\rho}-2 D^{\lambda \rho} \Lambda_{, \lambda}\right) x_{, \rho} / 2 .
$$

Since $\Gamma_{2}$ is harmonic to $S, \Gamma_{1}$ is conjugate to $S$.

Let covariant differentiation with respect to the form

$$
p D_{\rho \sigma} d u^{\rho} d u^{\sigma}
$$


be denoted by a semicolon. We find readily that

$$
x_{; \alpha \beta}=x_{, \alpha \beta}-\left[T_{\alpha \beta}^{\rho}+\left(\delta_{\alpha}^{\rho} P_{, \beta}+\delta_{\beta}^{\rho} P_{, \alpha}-D_{\alpha \beta} D^{\rho \lambda} P_{, \lambda}\right) / 2\right] x_{, \rho}, \quad P=\log p .
$$

Hence

$$
D^{\rho \sigma} x_{i \rho \sigma} / 2=y-T^{\rho} x_{, \rho} / 2+P_{\rho \sigma} D^{\rho \sigma} x / 2 .
$$

It follows that the line joining $x$ to the point whose general coordinates are $D^{\rho \sigma} x_{; \rho \sigma} / 2$ is the reciprocal of the line joining the points $x_{, 1}, x_{, 2}$ for every value of $p$.

Consider now the line $l_{1}$ joining $x$ to $y$. A point $z$ on $l_{1}$ has coordinates $z$ given by the formula

$$
z=y-\phi x .
$$

As $x$ moves along the $A$-curve the point $z$ describes a curve, a point $z^{\prime}$ on whose tangent has coordinates given by

$$
z^{\prime}=\left[\left(M_{\sigma}^{\rho}-\phi \delta_{\sigma}^{\rho}\right) x_{, \rho}+\left(Q_{\sigma}-\phi_{, \sigma}-\phi E_{\sigma}\right) x\right] A^{\sigma} .
$$

From (2.4) we may show that

$$
x_{, \alpha}=\left[B_{\alpha} X+A_{\alpha} Y-\left(A B_{\alpha}+B A_{\alpha}\right) x\right] / 2 .
$$

Hence we may write the coordinates of $z^{\prime}$ in the form

$$
z^{\prime}=A^{\sigma}\left(M_{\sigma}^{\rho}-\phi \delta_{\sigma}^{\rho}\right)\left(B_{\rho} X+A_{\rho} Y\right) / 2+() x,
$$

the coefficient of $x$ not being necessary for our purposes. It follows that the tangent to the locus of $z$ intersects the $B$-tangent at $x$ if and only if $\phi=\phi_{1}$ where

$$
\phi_{1}=M_{\sigma}^{\rho} D_{\rho \lambda} A^{\sigma} B^{\lambda} / 2=\left(M_{\rho}^{\rho}+M_{\sigma}^{\rho} D_{\rho \lambda} I^{\sigma \lambda}\right) / 2 .
$$

Interchanging the roles of the asymptotic tangents and curves on $S$, we find a second point determined by (2.8) with $\phi=\phi_{2}$ where

$$
\phi_{2}=\left(M_{\rho}^{\rho}+M_{\sigma}^{\rho} D_{\rho \lambda} I^{\lambda \sigma}\right) / 2 .
$$

The two points $z_{1}, z_{2}$ determined by $\phi_{1}, \phi_{2}$ coincide if and only if

$$
\left(M_{\sigma}^{\rho} D_{\rho \lambda}-M_{\lambda}^{\rho} D_{\rho \sigma}\right) I^{\sigma \lambda}=0,
$$

that is, if and only if

$$
E_{\alpha, \beta}-E_{\beta, \alpha}=0 .
$$

Hence on an arbitrary line $l_{1}$ protruding from $S$ at $x$ there exist two points $z_{1}, z_{2}$, which, as $x$ moves along respectively the $A$-curve and $B$-curve, describe curves whose tangents intersect respectively the $B$-tangent and $A$-tangent. These points coincide if and only if the congruence $\Gamma_{1}$ is conjugate to the surface.

We conclude this section by deriving the condition that the surface $S$ be 
ruled. The osculating plane at $x$ to a curve $C$ through $x$ whose tangent is determined by the contravariant components $C^{p}$ passes through the three points

$$
x, \quad C^{\rho} x_{, \rho}, \quad D_{\rho \sigma} C^{\rho} C^{\sigma} y+C_{, \sigma}^{\rho} C^{\sigma} x_{, \rho} .
$$

These points are collinear, that is $C$ is a straight line, if and only if $C$ is an asymptotic curve, say an $A$-curve, and if and only if

$$
R_{1}=2 A_{\rho} A_{, \sigma}^{\rho} A^{\sigma}
$$

vanishes. In a similar manner the $B$-curve is a straight line if and only if

$$
R_{2}=2 B_{\rho} B_{, \sigma}^{\rho} B^{\sigma}
$$

vanishes. Hence the surface $S$ is ruled if and only if the invariant $R=R_{1} R_{2}$ vanishes. Using (2.1), (2.2) and (2.3) we may write $R$ in the form

$$
R=D_{\rho \lambda, \sigma} D_{\phi \mu, \theta}\left(I^{\rho \phi} I^{\lambda \mu} D^{\sigma \theta}+I^{\rho \phi} I^{\sigma \theta} D^{\lambda \mu}+I^{\lambda \mu} I^{\sigma \theta} D^{\rho \phi}+D^{\lambda \mu} D^{\rho \phi} D^{\sigma \theta}\right) .
$$

3. The quadrics of Darboux. By proper choice of unit point, the point $Y$ whose general coordinates are given by the expression

$$
Y=y^{0} x+y^{p} x, y^{\prime}+y^{3} y
$$

will have local coordinates $\left(y^{0}, y^{1}, y^{2}, y^{3}\right)$ referred to the tetrahedron $\left(x, x_{1,1}\right.$, $\left.x_{, 2}, y\right)$. In terms of these local coordinates the equations of $S$ may be written in the form

$$
\begin{aligned}
& y^{0}=1+P_{\rho \sigma} \Delta u^{\rho} \Delta u^{\sigma} / 2+\cdots, \\
& y^{\alpha}=\Delta u^{\alpha}+L_{\rho \sigma}^{\alpha} \Delta u^{\rho} \Delta u^{\sigma} / 2+\cdots, \\
& y^{3}=D_{\rho \sigma} \Delta u^{\rho} \Delta u^{\sigma} / 2+\Sigma_{(\rho \sigma \lambda)} \Delta u^{\rho} \Delta u^{\sigma} \Delta u^{\lambda} / 6+\cdots,
\end{aligned} \quad \alpha=1,2,
$$

wherein

$$
\begin{aligned}
3 \Sigma_{(\rho \sigma \lambda)}= & D_{\rho \sigma, \lambda}+D_{\sigma \lambda, \rho}+D_{\lambda \rho, \sigma}+D_{\alpha \beta} E_{\gamma}+D_{\beta \gamma} E_{\alpha}+D_{\gamma \alpha} E_{\beta} \\
& +3\left(D_{\rho \mu} L_{\sigma \lambda}^{\mu}+D_{\sigma \mu} L_{\rho \lambda}^{\mu}+D_{\lambda \mu} L_{\rho \sigma}^{\mu}\right) .
\end{aligned}
$$

From (3.1) we may show that each of the quadrics,

$$
D_{\rho \sigma} y^{\rho} y^{\sigma}+y^{3}\left(-2 y^{0}+k_{\rho} y^{\rho}+k_{3} y^{3}\right)=0,
$$

has second order contact with $S$ at $x$ for arbitrary values of $k_{1}, k_{2}, k_{3}$. The triple point tangents of the curve of intersection of $S$ and a quadric of the family (3.2) are given by the expression

$$
\pi_{\rho \sigma \lambda} d u^{\rho} d u^{\sigma} d u^{\lambda}=0,
$$

wherein

$$
3 \pi_{\rho \sigma \lambda}=D_{\rho \sigma, \lambda}+D_{\sigma \lambda, \rho}+D_{\lambda \rho, \sigma}+D_{\rho \sigma} l_{\lambda}+D_{\sigma \lambda} l_{\rho}+D_{\lambda \rho} l_{\sigma}, \quad l_{\alpha}=E_{\alpha}-3 k_{\alpha} / 2 .
$$


The forms appearing in the left members of (1.7) and (3.3) are apolar if and only if

$$
\begin{aligned}
3 D^{\rho \sigma} \pi_{\rho \sigma \alpha} & =4\left(\Delta_{\alpha}+l_{\alpha}\right)=0, \\
4 \Delta_{\alpha} & =D^{\rho \sigma}\left(2 D_{\rho \alpha, \sigma}+D_{\rho \sigma, \alpha}\right) .
\end{aligned}
$$

Hence $k_{\alpha}=T_{\alpha}$. It follows therefore that the quadrics of Darboux have the equations

$$
D_{\rho \sigma} y^{\rho} y^{\sigma}+y^{3}\left(-2 y^{0}+T_{\rho} y^{\rho}+k_{3} y^{3}\right)=0,
$$

$k_{3}$ being an arbitrary parameter.

The differential equation of the curves of Darboux may be written in the form

$$
P_{\rho \sigma \lambda} d u^{\rho} d u^{\sigma} d u^{\lambda}=0
$$

wherein

$$
3 P_{\rho \sigma \lambda}=D_{\rho \sigma, \lambda}+D_{\sigma \lambda, \rho}+D_{\lambda \rho, \sigma}-\left(D_{\rho \sigma} \Delta_{\lambda}+D_{\sigma \lambda} \Delta_{\rho}+D_{\lambda \rho} \Delta_{\sigma}\right) .
$$

4. On conjugate nets. Let the contravariant components of the asymptotic tangent vectors be again denoted by $A^{\alpha}, B^{\alpha}$ and subject to the conditions (2.1). Let $U^{\alpha}, V^{\alpha}$ be the contravariant components of two other distinct tangent vectors. We may write these latter components in the form

$$
U^{\rho}=m A^{\rho}+n B^{\rho}, \quad V^{\rho}=p A^{\rho}+q B^{\rho}, . \quad m q-n p \neq 0 .
$$

We find readily that

$$
D_{\rho \sigma} U^{\rho} V^{\sigma}=2(m q+n p) .
$$

We shall speak of the curves whose tangent vectors are $U^{\alpha}, V^{\alpha}$ as the $U$ curves and $V$-curves respectively, with similar names for the tangents to these curves. We see readily that the $U$-tangents and $V$-tangents are conjugate if and only if $m q+n p=0$. Hence the contravariant components of conjugate tangent vectors may be written in the form

$$
U^{\rho}=\lambda\left(m A^{\rho}+n B^{\rho}\right), \quad V^{\rho}=\mu\left(m A^{\rho}-n B^{\rho}\right), \quad \lambda \mu m n \neq 0 .
$$

We find readily that

$$
D_{\rho \sigma} U^{\rho} U^{\sigma}=4 \lambda^{2} m n, \quad D_{\rho \sigma} V^{\rho} V^{\sigma}=-4 \mu^{2} m n .
$$

We may therefore choose $\lambda, \mu$ so that $m n=1 / 4$. Hence the contravariant components of conjugate tangent vectors may be written in the form

$$
U^{\rho}=m A^{\rho}+n B^{\rho}, \quad V^{\rho}=m A^{\rho}-n B^{\rho}, \quad 4 m n=1 .
$$

It follows that

$$
D_{\rho \sigma} U^{\rho} U^{\sigma}=1, \quad D_{\rho \sigma} V^{\rho} V^{\sigma}=-1 .
$$

Any point $X$ on the $U$-tangent has coordinates of the form

$$
X=U^{\rho} x_{, \rho}+U x .
$$


A point $X^{\prime}$ on the tangent at $X$ to the locus of that point as $x$ moves along the $V$-curve through $x$ has coordinates

$$
X^{\prime}=\left(U_{, \rho}^{\rho} V^{\sigma}+U V^{\rho}+\lambda U^{\rho}\right) x_{, \rho}+() x .
$$

The point $X^{\prime}$ is on the $U$-tangent if and only if

$$
U V^{\rho}+\lambda U^{\rho}=-U_{,{ }_{0}}^{\rho} V^{\sigma} \text {. }
$$

Hence the focal point $X$ on the $U$-tangent has coordinates defined by the expression

$$
X=U^{\rho} x_{, \rho}+V_{\rho} U_{, \sigma}^{\rho} V^{\sigma} x .
$$

Similarly the focal point $Y$ on the $V$-tangent through $x$ has coordinates given by the formula

$$
Y=V^{\rho} x_{, \rho}-U_{\rho} V_{, \sigma}^{\rho} U^{\sigma} x .
$$

From (4.2) and (4.3) we find readily that the ray of the conjugate net joins the points $R_{\alpha}$ whose general coordinates are given by the expression

$$
R_{\alpha}=x_{, \alpha}+\lambda_{\alpha} x
$$

wherein

$$
\lambda_{\alpha}=-\left(V_{\alpha} U_{\rho, \sigma} V^{\rho} U^{\sigma}+U_{\alpha} V_{\rho, \sigma} U^{\rho} V^{\sigma}\right) .
$$

It is easy to show that the contravariant components $\bar{U}^{\alpha}, \bar{V}^{\alpha}$. of any other conjugate vectors may be written in the form

$$
\begin{aligned}
& \bar{U}^{\rho}=U^{\rho} \cosh H+V^{\rho} \sinh H, \\
& \bar{V}^{\rho}=U^{\rho} \sinh H+V^{\rho} \cosh H,
\end{aligned}
$$

and that the $\bar{U}$-curves and $\bar{V}$-curves form a pencil [11] of conjugate nets if $H$ is constant. In particular the associate conjugate net of the given conjugate net is given by (4.4) with $H=i \pi / 4$.

Using (4.2) and (4.4) we easily show that the focal point $X$ on the tangent to the $\bar{U}$-curve has coordinates given by the formula

(4.5) $\bar{X}=X \cosh H+Y \sinh H+\sinh H \cosh H(\Phi \sinh H-\Psi \cosh H)$, wherein

$$
\begin{aligned}
& \Phi=U_{\rho}\left(U_{. \sigma}^{\rho} U^{\sigma}+V_{, \sigma}^{\rho} V^{\sigma}\right)+V_{\rho}\left(U_{, \sigma}^{\rho} V^{\sigma}+V_{.,}^{\rho} U^{\sigma}\right), \\
& \Psi=-\left[V_{\rho}\left(U_{, \sigma}^{\rho} U^{\sigma}+V_{, \sigma}^{\rho} V^{\sigma}\right)+U_{\rho}\left(U_{, \sigma}^{\rho} V^{\sigma}+V_{, \sigma}^{\rho} U^{\sigma}\right)\right] .
\end{aligned}
$$

Or using (2.10) and (2.11) we may write (4.6) in the form

$$
\Phi=\left(m^{3} R_{1}+n^{3} R_{2}\right) / 4, \quad \Psi=-\left(m^{3} R_{1}-n^{3} R_{2}\right) / 4 .
$$

Hence 


$$
R=\left(\Phi^{2}-\Psi^{2}\right) / 4 \text {. }
$$

Setting $H=i \pi / 4$ in (4.5) we find that the associate ray joins the points $\bar{R}_{1}, \bar{R}_{2}$ where

$$
\bar{R}_{\alpha}=x_{, \alpha}+\bar{\lambda}_{\alpha} x,
$$

wherein

$$
\bar{\lambda}_{\alpha}=\lambda_{\alpha}+\left(\Phi U_{\alpha}-\Psi V_{\alpha}\right) / 2 \text {. }
$$

Let us now consider the axis of the given net. From (2.9) the osculating plane of the $U$-curve at $x$ is determined by the points

$$
x, \quad U^{\rho} x_{, \rho}, \quad y+U_{, \rho}^{\rho} U^{\rho} x_{, \rho} .
$$

If we write the general coordinates of a point $Z$ in the form

$$
Z=x_{1} x+x_{2} X+x_{3} Y+x_{4} y
$$

the equation of this osculating plane in local coordinates, referred to the tetrahedron $(x, X, Y, y)$, is

$$
x_{3}+V_{\rho} U_{, \sigma}^{\rho} U^{\sigma} x_{4}=0 .
$$

Similarly the osculating plane at $x$ to the $V$-curve through $x$ has the equation

$$
x_{2}+U_{\rho} V_{, \sigma}^{\rho} V^{\sigma} x_{4}=0 .
$$

These osculating planes intersect in the point $x$ and in the point $Z$ whose general coordinates are given by the expression

$$
Z=y-\theta^{\rho} x_{:}
$$

wherein

$$
\theta^{\rho}=U_{\lambda} V_{, \sigma}^{\lambda} V^{\sigma} U^{\rho}+V_{\lambda} U_{,{ }_{,}}^{\lambda} U^{\sigma} V^{\rho} .
$$

The axis of any net of the pencil (4.4) joins $x$ to the point $\bar{Z}$ whose general coordinates are given by the formula

$$
\begin{aligned}
\bar{Z}= & \left(\bar{U}_{\rho} \bar{V}_{,{ }_{\sigma}} \bar{V}^{\sigma} \cosh H+\bar{V}_{\rho} \bar{U}_{,{ }_{\sigma}} \bar{U}^{\sigma} \sinh H\right) X \\
& +\left(\bar{U}_{\rho} \bar{V}_{,{ }_{\sigma}} \bar{V}^{\sigma} \sinh H+\bar{V}_{\rho} \bar{U}_{,{ }_{\sigma}} \bar{U}^{\sigma} \cosh H\right) Y-y .
\end{aligned}
$$

The equations of the axis of the net (4.4) may be written in local coordinates in the form

$$
\begin{aligned}
& x_{2}+\left[U_{\rho} V_{, \sigma}^{\rho} V^{\sigma}+\sinh 2 H(\Phi \sinh 2 H-\Psi \cosh 2 H) / 2\right] x_{4}=0, \\
& x_{3}+\left[V_{\rho} U_{, \sigma}^{\rho} U^{\sigma}+\sinh 2 H(\Phi \cosh 2 H-\Psi \sinh 2 H) / 2\right] x_{4}=0 .
\end{aligned}
$$

It follows therefore that the axes of all nets of a pencil of conjugate nets on a quadric surface coincide with the axis of the given net. Moreover if the surface 
is a non-quadric ruled surface, the locus of the axis of a net of a pencil of conjugate nets is a plane. The equation of this plane is

$$
\Phi y_{2}+\Psi y_{3}=0
$$

wherein

$$
\begin{aligned}
& y_{2}=x_{2}+U_{\rho} V_{, \sigma}^{\rho} V^{\sigma} x_{4}, \\
& y_{3}=x_{3}+V_{\rho} U_{, \sigma}^{\rho} U^{\sigma} x_{4} .
\end{aligned}
$$

If $S$ is not ruled, homogeneous elimination of $H$ from (4.9) yields the following equation of the axis quadric cone [10]:

$$
y_{2}^{2}-y_{3}^{2}-\left(\Phi y_{2}+\Psi y_{3}\right) x_{4} / 2=0 \text {. }
$$

The tangent plane to this cone along the axis of the given conjugate net has the equation

$$
\Phi y_{2}+\Psi y_{3}=0 \text {. }
$$

This tangent plane intersects the tangent plane to $S$ at $x$ in the second canonical tangent of the given conjugate net.

Setting $H=\pi i / 4$ in (4.4) we find that the associate axis joins $x$ to the point whose general coordinates are

$$
y-\bar{\theta}^{\rho} x, \rho
$$

wherein

$$
\bar{\theta}^{\rho}=\theta^{\rho}-\left(\Phi U^{\rho}-\Psi V^{\rho}\right) / 2 .
$$

From (4.7) and (4.10) we verify Green's theorem [4] that the ray and associate ray congruence of a conjugate net coincide if and only if the axis and associate axis congruence coincide; the sustaining surface must be a quadric.

Consider now a point $Z$ on the line $l_{1}$ joining the points $x, y$. The coordinates of $Z$ are of the form

$$
Z=y-\phi x .
$$

As $x$ moves along the $U$-curve of the conjugate net the point $Z$ moves on a curve: A point $Z^{\prime}$ on this latter curve has coordinates

$$
Z^{\prime}=\left(M_{\sigma}^{\rho}-\phi \delta_{\sigma}^{\rho}\right)\left(U_{\rho} X-V_{\rho} Y\right) U^{\sigma}+() x .
$$

The tangent line to the locus of $Z$ intersects the $V$-tangent at $x$ if and only if $\phi=\phi_{1}$ where

$$
\phi_{1}=-M_{\sigma}^{\rho} D_{\rho \lambda} U^{\sigma} U^{\lambda} .
$$

Interchanging the roles of the curves of the net, we find a second point defined by (4.11) with $\phi=\phi_{2}$ where 


$$
\phi_{2}=M_{\sigma}^{\rho} D_{\rho \lambda} V^{\sigma} V^{\lambda} .
$$

We shall speak of the two points $Z$ defined by (4.11) with $\phi=\phi_{1}$ and $\phi=\phi_{2}$ as the involutory points on $l_{1}$ with respect to the conjugate net. Using (4.1), we may write (4.12), (4.13) in the form

$$
\begin{aligned}
& \phi_{1}=M_{\rho}^{\rho} / 2+\left(m^{2} P+n^{2} Q\right), \\
& \phi_{2}=M_{\rho}^{\rho} / 2-\left(m^{2} P+n^{2} Q\right),
\end{aligned}
$$

wherein

$$
P=M_{\sigma}^{\rho} D_{\rho \lambda} A^{\sigma} A^{\lambda}, \quad Q=M_{\sigma}^{\rho} D_{\rho \lambda} B^{\sigma} B^{\lambda} .
$$

Using (1.5) and (2.3) we may show the vanishing of $P$ and $Q$ implies that the developables of the congruence $\Gamma_{1}$ of lines $l_{1}$ intersect $S$ in the asymptotic net on $S$. The unique conjugate net defined by (4.1) with

$$
m^{2} P+n^{2} Q=0, \quad P Q \neq 0
$$

has coincident involutory points on $l_{1}$.

If the developables of the congruence $\Gamma_{1}$ do not intersect $S$ in the asymptotic curves, then the involutory points on $l_{1}$ form an involution as the generating conjugate net varies through the conjugate nets on $S$. The double points of this involution are the point $x$ and the $K$-point of $x$ on $l_{1}$.

5. On canonical forms. The transformation

$$
x=\mu \bar{x}, \quad \mu \neq 0, \quad \log \mu=U,
$$

transforms (1.3) into the system

$$
\begin{aligned}
\bar{x}_{; \alpha \beta} & =\bar{P}_{\alpha \beta} \bar{x}+\bar{D}_{\alpha \beta} y, \\
y_{; \alpha} & =\bar{M}_{\alpha}^{\rho} \bar{x}_{; \rho}+\bar{Q}_{\alpha} \bar{x}+\bar{E}_{\alpha} y
\end{aligned}
$$

wherein the semicolon denotes covariant differentiation with respect to the transformed connection

$$
\bar{L}_{\alpha \beta}^{\rho}=L_{\alpha \beta}^{\rho}-\delta_{\alpha}^{\rho} U_{, \beta}-\delta_{\beta}^{\rho} U_{, \alpha},
$$

and wherein

$$
\begin{gathered}
\bar{P}_{\alpha \beta}=P_{\alpha \beta}-U_{, \alpha \beta}-U_{, \alpha} U_{, \beta}, \\
\mu \bar{D}_{\alpha \beta}=D_{\alpha \beta}, \quad \bar{M}_{\alpha}^{\rho}=\mu M_{\alpha}^{\rho}, \\
\bar{Q}_{\alpha}=\mu\left(Q_{\alpha}+M_{\alpha}^{\rho} U_{, \rho}\right), \quad \bar{E}_{\alpha}=E_{\alpha .} .
\end{gathered}
$$

Let $R$ be a function having the following properties (P): (a) $R \neq 0$, (b) $R$ is absolutely invariant under (1.2), (c) the transform $\bar{R}$ of $R$ under (1.9) is given by $a \bar{R}=R$, and by (d) $\bar{R}=\mu R$ under (5.1). We easily verify that the function $D$ defined by (1.6) transforms according to the laws $\bar{D}=a^{2} D$ under (1.11) and $\bar{D}=\mu^{-2} D$ under (5.1). 
The coordinates of the point $r_{\alpha}$ defined by the formula

$$
r_{\alpha}=x_{, \alpha}-f_{\alpha} x / 4,
$$

wherein

$$
f_{\alpha}=L_{\rho \alpha}^{\rho}+E_{\alpha}-2(\log R)_{, \alpha}-(\log D)_{, \alpha} / 2,
$$

transform under (1.9) and (5.1) according to the respective laws of transformation $\bar{r}_{\alpha}=r_{\alpha}, \bar{r}_{\alpha}=\mu r_{\alpha}$, and are therefore the covariant components of a vector. Moreover under the transformations (1.9) and (5.1) the transform $\bar{f}_{\alpha}$ of $f_{\alpha}$ is given by the equation

$$
\bar{f}_{\alpha}=f_{\alpha}-4 U_{, \alpha}
$$

Moreover $f_{\alpha, \beta}-f_{\beta, \alpha}=0$, and hence we may choose $\mu$ so that $f_{\alpha}=0$. The coordinates of the points $r_{\alpha}$ than assume the form

$$
r_{\alpha}=x_{, \alpha} .
$$

The form of (5.3) is preserved under (1.9) and (5.1) with $a$ arbitrary and $\mu=$ const.

The line joining $r_{1}$ and $r_{2}$ is an intrinsic line which we have previously called an $R$-harmonic line [7]. If in particular the invariant $R$ is taken as that invariant defined by (2.12) the line is the reciprocal of the Fubini-Green projective normal.

Let us assume that the coordinates $x$ are so normalized that the line joining $x_{1}, x_{, 2}$ is an $R$-harmonic line. According to (2.6) the reciprocal of the $R$ harmonic line joins $x$ to the point $Z$ given by

$$
Z=y-T^{\rho} x_{, \rho} / 2 \text {. }
$$

Under the transformation (1.9) the functions $T^{p}$ transform according to the law

$$
\bar{T}^{\rho}=a^{-1}\left(T^{\rho}-2 \theta^{\rho}\right) .
$$

Hence we may choose $\theta^{\rho}$ so that $\bar{T}^{\rho}=0$. We shall suppose that this transformation has been effected.

Since the line $x y$ is the reciprocal of an $R$-harmonic line, it is an $R$-conjugate line and the congruence generated by it is conjugate to $S$. It follows that condition (1.8) is fulfilled. The coefficient $a$ in the transformation

$$
y=\phi x+a \bar{y}
$$

may be chosen so that $\vec{E}_{\alpha}=0$; the coefficient $\phi$ in (5.4) may be chosen to make $\bar{M}_{\rho}^{\rho}=0$.

Hence we may reduce the system (1.3) to the form

$$
\begin{aligned}
x_{, \alpha \beta} & =P_{\alpha \beta} x+D_{\alpha \beta} y, \\
y_{, \alpha} & =M_{\alpha}^{\rho} x_{, \rho}+Q_{\alpha} x,
\end{aligned}
$$


in which the line joining $x_{, 1}, x_{, 2}$ is an $R$-harmonic line, $x y$ is the corresponding $R$-conjugate line, the point $y$ is the $K$-point on $x y$. The system is characterized analytically by the conditions

$$
f_{\alpha}=0, \quad T^{\alpha}=0, \quad E_{\alpha}=0, \quad M_{p}^{\rho}=0 .
$$

Suppose again for the moment that the defining differential equations are in the unspecialized form (1.3). Let there be given a form

$$
a_{\rho \sigma} d u^{\rho} d u^{\sigma}=0 \text {, }
$$

and let $\Gamma_{\rho \sigma}^{\alpha}$ be the Christoffel symbols formed with respect to the components $a_{\rho \sigma}$. We may readily show that

$$
\Gamma_{\rho \sigma}^{\alpha}=L_{\rho \sigma}^{\alpha}+A_{\rho \sigma}^{\alpha},
$$

wherein

$$
A_{\rho \sigma}^{\alpha}=a^{\alpha \lambda}\left(a_{\rho \lambda, \sigma}+a_{\sigma \lambda, \rho}-a_{\rho \sigma, \lambda}\right) / 2, \quad a^{\alpha \rho} a_{\rho \beta}=\delta_{\beta}^{\alpha} .
$$

Let a curve $C$ through $x$ be considered as imbedded in a one-parameter family of curves defined by the differential equation

$$
d u^{1}: d u^{2}=C^{1}: C^{2} .
$$

The curve $C$ is an extremal of the integral

$$
\int\left(a_{\rho \sigma} d u^{\rho} d u^{\sigma}\right)^{1 / 2}
$$

if the contravariant components $C^{\alpha}$ satisfy the condition

$$
\left[C^{\alpha} C_{, \lambda}^{\beta}-C^{\beta} C_{, \lambda}^{\alpha}+C^{\mu}\left(C^{\alpha} A_{\mu \lambda}^{\beta}-C^{\beta} A_{\mu \lambda}^{\alpha}\right)\right] C^{\lambda}=0 .
$$

Referred to the tetrahedron $x, x_{1,}, x_{, 2}, y$ the osculating plane at $x$ to the curve $C$ subject to the condition (5.5) has coordinates given by the formulas

$$
\begin{aligned}
\xi_{0}=0, & \xi_{1}=C^{2} D_{\rho \sigma} C^{\rho} C^{\sigma}, \quad \xi_{2}=-C^{1} D_{\rho \sigma} C^{\rho} C^{\sigma}, \\
\xi_{3} & =\left(C^{2} A_{\rho \sigma}^{1}-C^{1} A_{\rho \sigma}^{2}\right) C^{\rho} C^{\sigma} .
\end{aligned}
$$

Homogeneous elimination of $C^{\alpha}$ yields the following equation of the envelope of these osculating planes:

$$
4 D^{\rho \sigma} \xi_{\rho} \xi_{\sigma} l=Q^{\rho \sigma \lambda} \xi_{\rho} \xi_{\sigma} \xi_{\lambda}, \quad \xi_{0}=0,
$$

wherein

$$
l=\left(4 \xi_{3}-\left(3 D^{\rho \sigma} A_{\rho \sigma}^{\lambda}-2 D^{\lambda \sigma} A_{\rho \sigma}^{\rho}\right) \xi_{\lambda},\right.
$$

and wherein the coefficients $Q^{\rho \sigma \lambda}$ are immaterial for the present. It is easily shown that the cusp axis of the cone (5.6) has the equation $\xi_{0}=l=0$. 
This line joins the point $x$ to the point $Z$ whose general coordinates are given by the formula

$$
Z=y-\left(3 D^{\rho \sigma} A_{\rho \sigma}^{\lambda}-2 D^{\lambda \sigma} A_{\rho \sigma}^{\rho}\right) x, \lambda / 4 .
$$

From (2.7) the reciprocal of this cusp axis joins the points $r_{\alpha}$ defined by the formula

$$
r_{\alpha}=x_{, \alpha}+\left[T_{\alpha}-D_{\alpha \lambda}\left(3 D^{\rho \sigma} A_{\rho \sigma}^{\lambda}-2 D^{\lambda \sigma} A_{\rho \sigma}^{\rho}\right) / 4\right] x / 2 .
$$

If in particular we let $a_{\alpha \beta}=R D_{\alpha \beta}$ wherein $R$ has the properties (P), then the expressions (5.7) may be reduced to

$$
r_{\alpha}=x_{, \alpha}-f_{\alpha} x / 4,
$$

$f_{\alpha}$ being defined by (5.2). It follows that the cusp axis [1] of the extremals of the integral

$$
\int\left(R D_{\rho \sigma} d u^{\rho} d u^{\sigma}\right)^{1 / 2}
$$

is the $R$-conjugate line defined by the invariant $R$. If $R$ is the invariant (2.12), the cusp axis is the projective normal.

6. Metrical considerations. Let us specialize the differential equations (1.3) to be the Gauss differential equations of a surface immersed in euclidean space of three dimensions. We may interpret the functions $y$ as the direction cosines of the metric normal, and the comma as indicating covariant differentiation with respect to the metric tensor $g_{\alpha \beta}$.

Under these restrictions we may write the components of some of. the tensors in the previous theory in the following forms:

$$
T^{\alpha}=D^{\alpha \lambda}\left(\partial \log K / \partial u^{\lambda}\right) / 2, \quad f_{\alpha}=-\partial \log \left(K^{1 / 2} R^{2}\right) / \partial u^{\alpha},
$$

wherein $R$ is an absolute invariant under (1.2) and $K$ is the Gaussian curvature of the surface at $x$.

It follows from (2.6) that the reciprocal of the ideal line in the tangent plane to $S$ at $x$ has direction cosines proportional to

$$
y-D^{\rho \lambda}\left(\partial \log K x_{, \rho} / \partial u^{\lambda}\right) / 4 .
$$

Hence the reciprocal of the ideal line coincides with the metric normal if and only if the surface has constant Gaussian curvature. Or at the point $x$ on a surface of constant Gaussian curvature every quadric of Darboux cuts the metric normal orthogonally.

For surfaces of non-constant Gaussian curvature, the reciprocal of the metric normal joins the points $r_{\alpha}$ whose coordinates are given by the formula

$$
r_{\alpha}=x+\frac{2 x_{, \alpha}}{\partial \log K / \partial u^{\alpha}} \quad(\text { not summed on } \alpha) \text {. }
$$


Let $R$ be an absolute invariant under (1.2). Then the $R$-conjugate line has direction cosines proportional to the expressions

$$
y-\left(T^{\rho}+D^{\rho \sigma} f_{\sigma} / 2\right) x_{, \rho} / 2 .
$$

Using (6.1) we may write (6.2) in the form

$$
y+\frac{1}{4} D^{\rho \lambda} \frac{\partial}{\partial u^{\lambda}} \log \left(\frac{R^{2}}{K^{1 / 2}}\right) x_{, \rho} .
$$

It follows that the metric normal [2] is the special $R$-conjugate line for

$$
R=c K^{1 / 4}
$$

(c a nonzero constant).

In particular if the function $R$ is that function defined by (2.12), the condition (6.3) is a necessary and sufficient condition that the projective normal and the metric normal coincide [9].

Let the functions $\mathfrak{A}$ and $\mathfrak{B}$ be defined by the formulas

$$
\mathfrak{\Re}^{2}=g_{\rho \sigma} A^{\rho} A^{\sigma}, \quad \mathfrak{B}^{2}=g_{\rho \sigma} B^{\rho} B^{\sigma} .
$$

We may show that the lines of curvature are given by (4.1) with

$$
m=\mathfrak{A}, \quad n=\mathfrak{B} \text {. }
$$

Hence we may apply the formulas in $\$ 5$ to study the lines of curvature. We shall not enter into these discussions at present.

Finally let (1.3) be again interpreted as the definining differential equations of a surface in a projective space of three dimensions. Let the line $x y$ be chosen as an $R$-conjugate line with $R$ unspecialized. Let $y$ be an unspecialized point on $x y$. By virtue of (1.8) we may choose a multiplier of the functions $y$ so that $E_{\alpha}=0$. The last of equations (1.4) shows that there exists a symmetric tensor $G^{\alpha \beta}$ defined by the formula

$$
G^{\alpha \beta}=-D^{\alpha \rho} M_{\rho}^{\beta}=-D^{\beta \rho} M_{\rho}^{\alpha} .
$$

If $M \neq 0$ there exist covariant components $G_{\alpha \beta}$ defined uniquely by the formula

$$
G_{\alpha \rho} G^{\rho \beta}=\delta_{\alpha}^{\beta}
$$

We may easily show that

$$
G^{\sigma \lambda} M_{\sigma}^{\rho} I_{p \lambda}=0,
$$

that is, the curves corresponding to the developables of the $R$-conjugate congruence are "orthogonal" in the metric based on $G_{\alpha \beta}$.

Again from (1.4) and (6.4) one may show that

$$
P_{\delta \alpha \beta \gamma}=G_{\delta \lambda} P_{\alpha \beta \gamma}^{\lambda}=D_{\delta \beta} D_{\alpha \gamma}-D_{\delta \gamma} D_{\alpha \beta} \text {. }
$$


Hence

$$
P_{1212}=D
$$

Again from (6.4) we find that

$$
M=D / G, \quad G=\left|G_{\alpha \beta}\right| .
$$

We may call the tensor [8] $P_{\delta \alpha \beta \gamma}$ the projective curvature tensor of the surface $S$ relative to the $R$-conjugate line $x y$ and the point $y$.

For a given $R$-conjugate congruence and a particular choice of ideal point $y$ on the line of the congruence through $x$, the foregoing presents a natural manner of introducing a metric on the surface. We shall refrain from the elaboration of this theory for the present.

\section{REFERENCES}

1. P. O. Bell, Projective analogues of the congruence of normals, Amer. J. Math. vol. 52 (1940) pp. 680-686.

2. - On differential geometry intrinsically connected with a surface element of projective arc length, Trans. Amer. Math. Soc. vol. 50 (1941) pp. 529-547.

3. G. M. Green, On the theory of curved surfaces and canonical systems in projective differential geometry, Trans. Amer. Math. Soc. vol. 16 (1915) pp. 1-12.

4. - Projective differential geometry of one-parameter families of space curves, and coniugate nets on a curved surface, second memoir, Amer. J. Math. vol. 38 (1916) pp. 287-324.

5. - Memoir on the general theory of surfaces and rectilinear congruences, Trans. Amer. Math. Soc. vol. 20 (1919) pp. 80-153.

6. V. G. Grove, A canonical form of Green's projective analogue of the Gauss differential equations, Trans. Amer. Math. Soc. vol. 32 (1930) pp. 473-484.

7. - On canonical forms of differential equations, Bull. Amer. Math. Soc. vol. 36 (1930) pp. 582-586.

8. - A tensor analysis for a $V_{k}$ in a projective space $S_{n}$, Bull. Amer. Math. Soc. vol. 45 (1939) pp. 385-398.

9. E. P. Lane, Projective differential geometry of curves and surfaces, The University of Chicago Press, 1932. 1942.

10. - A treatise on projective differential geometry, The University of Chicago Press,

11. E. J. Wilczynski, Geometrical significance of isothermal conjugacy, Amer. J. Math. vol. 42 (1920) pp. 211-221.

Michigan State: College,

East Lansing, Mich. 